\title{
Modelling Freeway Networks by Hybrid Stochastic Models
}

\author{
René Boel \\ SYSTeMS, Universiteit Gent \\ B-9052 Zwijnaarde, Belgium \\ rene.boel@ugent.be
}

\author{
Lyudmila Mihaylova
}

SYSTeMS, Universiteit Gent
B-9052 Zwijnaarde, Belgium
mila.mihaylova@ieee.org

\begin{abstract}
Traffic flow on freeways is a nonlinear, many-particle phenomenon, with complex interactions between the vehicles. This paper presents a stochastic hybrid model of freeway traffic at a time scale and at a level of detail suitable for on-line flow estimation, for routing and ramp metering control. The model describes the evolution of continuous and discrete state variables. The freeway is considered as a network of components, each component representing a different section of the network. The traffic model, designed from physical considerations, comprises sending and receiving functions describing the downstream and upstream propagation of perturbations to be controlled. Results from simulation investigations illustrate the effectiveness of our model compared to the well-known METANET model.
\end{abstract}

\section{Motivation}

Traffic flow on freeways is a complex process and its online management incorporates many aspects: analysis of the traffic phenomena, building models of traffic dynamics and observations that are adequate for control purposes, development of cost effective and reliable data-processing algorithms for state estimation and control. A freeway network consists of many interacting components which can exhibit highly variable behavior, such as traffic jams, stop-and-go waves and hysteresis phenomena. Experimental studies [5] based on traffic data have shown that traffic flow in different sections of the freeway possesses distinct dynamic modes (phases) such as: free flow traffic where vehicles do not interact much with each other and where vehicles approach their own desired speed; synchronized traffic flow, a mode in which drivers move with nearly the same speed on the different lanes of the highway; congested mode in which the speed is quite low and can fluctuate very much while flow does not vary significantly; and jammed mode, where both speed and flow are very low. Some changes in the section mode, for example from "free flow" to "congested" are induced by the traffic dynamics themselves, for instance the drivers' behavior, while other transitions are due to external events such as accidents, road works or weather conditions. Perturbations are propagated from upstream to downstream sections via forward waves. Different phenomena are observed in jammed mode. Drivers are slowing down when they observe traffic congestion in the section ahead of them which increases the traffic density upstream provoking a backward wave. In [1], [2] for the first time linear and static sending and receiving functions are proposed to model these waves together with a cell-transmission model.

We represent the freeway traffic as a network of stochastic dynamic components, each component describing a different section of the network. The approach of traffic modelling as a network of hybrid components offers modularity and flexibility, it allows parallel processing which can reduce the computational load. As such, it brings the scalability potential of the decentralized architectures, adaptability and improves system predictability. It is applicable to both freeways and urban networks. Changes in the topology of the freeway network only require addition or deletion of a few components (local changes to the model). The traffic is a stochastic hybrid system, which means that each traffic section possesses continuous and discrete states, interacting with variables from neighboring sections. Continuous variables in a section are the average speed and the number of vehicles in the section. Modal (discrete) state variables describing the abrupt changes in the traffic regimes are the number of lanes, traffic modes, external conditions (weather, road works), controlled events (e.g. change of traffic lights, speed limitations). Continuous variables are stochastic due to the random behavior of drivers reacting to local events, modal states are random due to unexpected events.

The novelty of this paper lies in the derived stochastic hybrid model of the freeway traffic, well suited for online estimation. It represents both forward and backward waves through the mechanism of sending and receiving functions. The traffic dynamics is described in this model by macroscopic (aggregated) variables, i.e. in terms of collective vehicle dynamics and treating the traffic as a fluid flow. 
Various macroscopic models have been developed over the last 60 years for deterministic or stochastic evolution of the three main variables of aggregated traffic: flow, speed, and density (for recent surveys, see [3], [5], [6], [7], [4], [9]). The majority of the macroscopic models proposed in the literature are deterministic. Until now macroscopic models are predominantly used to simulate the traffic dynamics, less for traffic state estimation and control. Compared to the other types of models with higher levels of detail, like microscopic (particle-based) and mesoscopic (gas-kinetic) [5], the macroscopic (fluiddynamic) models are more suitable for on-line predictions of traffic states with real data and for traffic regimes detection and for incident detection. These predictions are then useful for model based predictive control.

The outline of the paper is as follows. Section II presents the framework for modelling the traffic flow as a stochastic hybrid system. The freeway network is divided into components and each component is described by its dynamic mode with aggregated traffic variables. In Section III a compositional model of a link is developed, a substantial part of which are sending and receiving functions. Section IV presents a comparison between simulation results obtained using the model introduced in this paper and a stochastic version of METANET. Finally, conclusions and ongoing research issues are highlighted.

\section{Traffic Modelled as a Stochastic Hybrid System}

A network of freeways can be divided into stretches, called links, typically between two on- and off- ramps. A link is composed by a sequence of sections, as indicated in Fig. 1. Each section corresponds to a stretch of a freeway where the behavior is fairly homogeneous (usually few hundred meters long). The traffic flow in a section is modelled as a stochastic hybrid system, with continuous and discrete states, interacting with states from neighboring sections. The global state of link $m$ at time $t_{k}$ is described by the vector $x_{k}=\left(x_{1, k}^{T}, x_{2, k}^{T}, \ldots, x_{n_{m}, k}^{T}\right)^{T}$, containing the local state vectors $x_{i, k}=\left(N_{i, k}, v_{i, k}\right)^{T}$ of the $n_{m}$ sections forming this link. $N_{i, k}$ is the number of vehicles present in section $i$ at time $t_{k}$, and $v_{i, k}$ is their average speed. The state vector $x_{k}$ is sampled at possibly asynchronous points in time $t_{1}<t_{2}<\ldots<t_{k}<\ldots$. The evolution from one sample time to the next sample time is described by the update equation

$$
x_{k+1}=f\left(x_{k}, \mathcal{P}_{k}, Q_{k}^{\text {in }}, v_{k}^{\text {in }}, Q_{k}^{\text {out }}, v_{k}^{\text {out }}, \eta_{k}\right)
$$

where $\mathcal{P}_{k}$ denotes the vector of all time-varying parameters, such as road conditions, or number of available lanes; $Q_{i n, k}$ counts the number of vehicles entering section 1 during the $k$-th time interval $\left[t_{k}, t_{k+1}\right)$ while $v_{i n, k}$ is the average speed of these vehicles. $Q_{o u t, k}$ specifies the possible outflow, at speed $v_{\text {out }, k}$ from section $n_{m} . \eta_{k}$ is a disturbance vector, reflecting random fluctuations in the traffic states, and modelling errors. The traffic mode in section $i$ in this interval can make sudden transitions between different modes with rates which depend on the state vectors $x_{i-1, k}, x_{i, k}, x_{i+1, k}$. Using traffic modes as discrete state variables allows for much simpler form of $f_{i}$ (e.g. affine function). In the first and the last zones of a section, $d_{i, k}$ acts as a part of boundary conditions. These first and last zones are special because they describe boundary conditions, including the interaction with upstream and downstream sections, on- and off-ramps, etc.

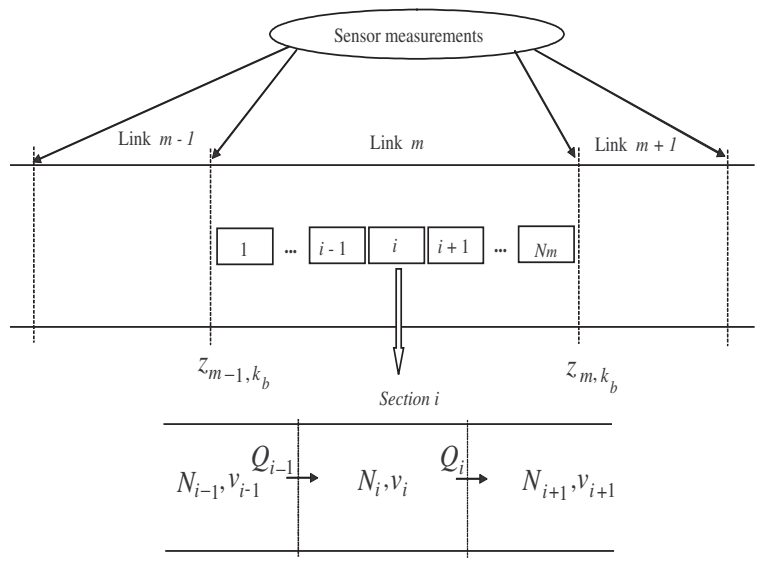

Fig. 1. Freeway links, sections and measurement points. $Q_{i}$ is the average number of vehicles at the boundary between sections $i$ and $i+1, N_{i}$ and $v_{i}$ are respectively the average number of vehicles and speed within section $i$.

The observation equation is of the form

$$
z_{i, k_{b+1}}=h_{i}\left(x_{i, s}, s \in\left[t_{k_{b}}, t_{k_{b+1}}\right), \xi_{i, k_{b+1}}\right),
$$

where the measurement vector $z_{i, k_{b}}=\left(\bar{Q}_{i, k_{b}}, \bar{v}_{i, k_{b}}\right)^{T}$ comprises the average number of vehicles (cars and trucks) $\bar{Q}_{i, k_{b}}$, crossing the boundary between section $i$ and section $i+1$ over the interval $\left[t_{k_{b}}, t_{k_{b+1}}\right.$ ) (only for the sections equipped by sensors). The different time indices in (2) emphasize that the data are aggregated over intervals $\left[t_{k_{b}}, t_{k_{b+1}}\right)$ at regular or irregular points in time, typically less frequent than the state update (or sample) times $t_{k}$.

The measurements are obtained by induction loop detectors or video cameras at the boundary between some sections (at least one sensor at entrance and exit). They are corrupted by noise due to sensor calibration errors, 
communication link errors, sensor failures. Sensor failures raise missing data problems which the traffic estimation/ control algorithm should be able to cope. Usually the data are received from non-equidistant points in space. Functions $f_{i}$ and $h_{i}$ are nonlinear in general and can be different in the different sections of the freeway. One form of the functions $f_{i}$ and $h_{i}$ is given in the next section, where a compositional model of a link is derived from physical considerations.

\section{Compositional model of a link}

Each section receives vehicles from the upstream section, and sends vehicles to the downstream section. Hence, the dynamic evolution of the traffic state of a link depends on the interaction between neighbor links, i.e. on the inflow and outflow at upstream and downstream boundary of the link.

\section{A. Updating the number of vehicles}

The number of vehicles, $N_{i, k}$, [veh], within a freeway section $i$ (with length $L_{i},[k m]$, and with $\ell_{i, k}$ number of lanes, at sample time instant $t_{k}$ is related to the density $\rho_{i, k}$ (number of vehicles per length unit, $[\mathrm{veh} / \mathrm{km} /$ lane $]$ ) via

$$
N_{i, k}=\rho_{i, k} L_{i} \ell_{i, k}, \quad i=1,2, \ldots N_{m} .
$$

The evolution of $N_{i, k}$ can be expressed by

$$
N_{i, k+1}=N_{i, k}+Q_{i-1, k}-Q_{i, k},
$$

where $Q_{i, k}$ is the number of vehicles, [veh], crossing the boundary between two sections (Fig. 1), leaving section $i$ and entering the section $i+1$ during the interval $\left[t_{k}, t_{k+1}\right)$. $Q_{i, k}$ is the minimum

$$
Q_{i, k}=\min \left(S_{i, k}, R_{i, k+1}\right),
$$

among the values computed from a sending function

$S_{i, k}=\max \left\{N_{i, k} \frac{v_{i, k} \Delta t_{k}}{L_{i}}+\eta_{S_{i}, k}, N_{i, k} \frac{v_{\text {out }, \min } \Delta t_{k}}{L_{i}}\right\}$

and a receiving function $R_{i, k+1} . S_{i, k}$ is a random variable expressing how many among $N_{i, k}$ vehicles in section $i$ at $k$ are at a distance less than $v_{i, k} \Delta t_{k}$ from the boundary between sections $i$ and $i+1$, where $\Delta t_{k}=t_{k+1}-t_{k}$ is the sampling interval.

When the density is low (corresponding to free-flow mode of traffic, i.e. when $N_{i, k} \leq \rho_{\text {crit }} L_{i, k} \ell_{i, k} ; \rho_{\text {crit }}$ denotes the critical density) the interaction between vehicles will be negligible and their location will be uniformly distributed over $L_{i} . S_{i, k}$ is then a binomial random variable with $N_{i, k}$ drawings, with probability of success $\left(v_{i, k} \Delta t_{k}\right) / L_{i}$. If the traffic is congested, vehicles will be approximately equidistantly spaced and the noise is small (relative to $S_{i, k}$ ) and approximately Gaussian (by the central limit theorem).

This formula for $S_{i, k}$ does not take into account what happens when a severe traffic jam causes stopped traffic at time $t_{k}$ in section $i$. The speed then drops to 0 and no vehicle would ever leave section $i$ after time $t_{k}$, even if the downstream section was empty. Hence, we have to impose a minimum outflow speed $v_{\text {out,min }}$ with which vehicles attempt to leave section $i$. This minimal outflow speed can only be realized if the downstream section is not too congested, which effect is taken into account by the receiving function. This outflow speed $v_{\text {out } \text { min }}$ is chosen so that $\rho_{\text {jam }} v_{\text {out }, \text { min }} \ell_{i, k}$ equals the empirically observed outflow from a traffic jam into an empty downstream section, a value that is typically significantly smaller than the maximum flow.

The receiving function

$$
R_{i, k+1}=N_{i+1, k}^{\max }+Q_{i+1, k}-N_{i+1, k},
$$

where

$$
N_{i+1, k}^{\max }=\left(L_{i+1} \ell_{i+1, k}\right) /\left(A_{\ell}+v_{i+1, k} t_{d}\right),
$$

gives the number of vehicles that can enter section $i+1$ at the next time instant $k+1, v_{i, k}$ is the average speed of vehicles inside section $i . N_{i+1, k}^{\max }$ characterizes the maximum number of vehicles within section $i+1$ at sample time $k$, assuming that the average space needed by a vehicle is its average length $A_{\ell}$ plus the distance travelled. $t_{d}$ is the minimum time distance between vehicles. The sending function corresponds to downstream propagation and can be recursively calculated from left to right, whereas the receiving function corresponds to upstream propagation and must be recursively calculated from right to the left.

Evidently, this requires the solution of a system with $n_{m}$ nonlinear algebraic equations (4)-(8). Sources of nonlinearity are the minimum operation in (5), the multiplication of $N_{i, k}$ and $v_{i, k}$ in (6), as well as the division by $v_{i+1, k}$ in (8). To prevent possible negative values of $R_{i, k+1}$ (7) when abrupt changes happen, it is imposed

$$
N_{i+1, k}=N_{i+1, k}^{\max }, \quad \text { if } \quad N_{i+1, k}^{\max }<N_{i+1, k} .
$$

In order to describe this complex traffic behavior, the sending function is calculated at first forward by assuming that $Q_{i, k}=S_{i, k}$ and substituting it in (4). With this "guessed" number of vehicles on borders, a guessed number of vehicles inside sections, a guessed density and a guessed speed are calculated. After that the receiving function is computed from section $n_{m}$ down to section 
1 and the condition that the number of vehicles leaving section $i$ cannot be larger than the number of vehicles that can be received by section $i+1$ is imposed as follows:

$$
\text { if } \begin{aligned}
R_{i, k+1}<S_{i, k}, \text { then } Q_{i, k}=R_{i, k+1} \\
\quad \Delta_{i, k}=S_{i, k}-R_{i, k+1}, N_{i, k+1}-\text { recalculated } .
\end{aligned}
$$

$Q_{i, k}$ is the number of vehicles which actually are succeeding to cross the boundary, whereas $\Delta_{i, k}=S_{i, k}-R_{i, k+1}$ is the number of vehicles which are forced to remain in section $i$ by slowing down. $\Delta_{i, k}$ is added to $N_{i, k+1}$. With this updated value of $N_{i, k+1}$, the previous values of the receiving function are recalculated in order to avoid "overflow" of sections with vehicles. This "backward validation" is repeated until $\Delta_{i, k}=0$ (see (10)) for all sections.

\section{B. Updating the average speed}

The speed $v_{i, k+1}$ is calculated via the steps :

$$
v_{i, k+1}^{\text {interm }}=\left\{\begin{array}{l}
{\left[v_{i-1, k} Q_{i-1, k}+v_{i, k}\left(N_{i, k}-Q_{i, k}\right)\right] / N_{i, k+1},} \\
\text { for } N_{i, k+1} \neq 0 \\
\\
v_{f}, \text { otherwise. }
\end{array}\right.
$$

$$
\begin{aligned}
& \rho_{i, k+1}^{\text {antic }}=\alpha \rho_{i, k+1}+(1-\alpha) \rho_{i+1, k+1}, \\
& v_{i, k+1}=\beta v_{i, k+1}^{\text {interm }}+(1-\beta) v^{e}\left(\rho_{i, k+1}^{\text {antic }}\right)+\eta_{v_{i}, k},
\end{aligned}
$$

where $v_{i, k+1}^{\text {interm }}$ is the intermediate speed taking convection into account as if all vehicles would maintain their speed constant; $\rho_{i, k+1}^{a n t i c}$ is the anticipated traffic density which drivers see at some distance in front of their vehicles, and $\rho_{i, k+1}$ is computed from (3) after $N_{i, k+1}, i=1, \ldots, n_{m}$ has been evaluated. The coefficients $\alpha$ and $\beta$ are in the range: $(0,1]$. Formulae (11) and (13) express the fact that drivers can not change their speed instantaneously due to inertia. Anticipation leads drivers to reach a weighted average density in downstream sections. The noise $\eta_{v_{i}, k}$ reflects speed irregularities due to the unpredictable behavior of the drivers and also modelling errors. It is assumed to be a Gaussian random process. The relative noise $\eta_{v_{i}, k} / v_{i, k}$ is small for jammed traffic and high for synchronized and congested traffic. The equilibrium speed $v^{e}\left(\rho_{i, k+1}^{a n t i c}\right)$ can be computed according to eq. (19) [7] using simpler affine functions $v_{e}\left(\rho_{i}\right)$ corresponding to different traffic modes.

Then (4) and (13) are the traffic state equations of the $i$-th section, with a state vector $x_{i, k}=\left(N_{i, k}, v_{i, k}\right)^{T}$ of a section $i$, and the state vector of a link is $x_{k}=\left(x_{1, k}^{T}, x_{2, k}^{T}, \ldots, x_{n_{m}, k}^{T}\right)^{T}$. The initial vector $x_{0}=\left(N_{1,0}, v_{1,0}, \ldots, N_{n_{m}, 0}, v_{n_{m}, 0}\right)^{T}$, and the boundary variables (for the first and last segment) are of primary importance. The number of vehicles entering the first section is upper bounded by the sending flow, i.e. $Q_{1,0}=S_{1,0}, Q_{n_{m}, 0}=R_{n_{m}, 0}$. The number of the vehicles leaving the last section is bounded by the maximum outflow $R_{n_{m}, 0}$.

\section{Congestion propagation}

Congestion occurs often in freeway traffic. Congestion is a traffic flow phenomenon when the travel demand approaches or exceeds capacity of the facility. Traffic demands vary significantly depending on the season of the year, the day of the week, and the time of the day. Major reasons for congestions are: reduced capacity due to accidents, work zones, weather conditions (as fog, snow, rain), special events.

When the density is high, the state of a section often is such that traffic flow is lower than the capacity (maximal flow). This inefficiency is represented in the model by $v^{e}$ and spreads upstream via the receiving function. The backward wave, which is created during congestion, is modelled through the receiving function in the compositional model, reflecting the fact that a cell cannot emit more vehicles than the neighbor cell can receive. In terms of densities, traffic is congested when the density exceeds its critical value $\rho_{\text {crit }}$. The critical density $\rho_{\text {crit }}$ has to be distinguished from the density in jam, $\rho_{j a m}$, characterizing the jammed mode.

Advantages of the model (4)-(13) are that it does not require many computations, nor parameter adaptation. Parameters which are of primary importance are the critical density and the number of lanes. The value of the critical density is crucial in order to distinguish the free-flow mode from the congested mode.

\section{Observation equations}

The following observation equations are derived in agreement with (2)

$$
\begin{aligned}
& z_{j, k_{b}+1}(1)=\bar{Q}_{j, k_{b+1}}+\xi_{Q_{j}, k_{b+1}}, \\
& z_{j, k_{b+1}}(2)=\bar{v}_{j, k_{b+1}}+\xi_{v_{j}, k_{b+1}},
\end{aligned}
$$

where $z_{j, k_{b}+1}(1)$ is the measured averaged number of vehicles crossing the boundary between sections $j$ and $j+1$ during the time interval $\Delta t_{b}=\left[t_{k_{b}}, t_{k_{b+1}}\right)$, namely $\bar{Q}_{j, k_{b+1}}=\frac{1}{\Delta t_{b}} \sum_{k=t_{b}}^{k=t_{b+1}} Q_{j, k}, j \in J \subset\left\{1, \ldots, n_{m}\right\} ;$ $z_{j, k_{b+1}}(2)$ is the average speed over the same interval of these $Q_{j, k}$ vehicles. Note that this average speed weighs 
the faster vehicles more heavily than the slower vehicles, since faster vehicles cross boundaries more frequently.

\section{A comparison to stochastic METANET model}

The compositional model introduced in Section III is validated by comparing simulation results of (4)-(13) with results obtained via results from a stochastic version of METANET model [8], [7]

$$
\begin{aligned}
& \rho_{i, k+1}=\rho_{i, k}+\frac{\Delta t_{k}}{L_{i} l_{i, k}}\left(q_{i-1, k}-q_{i, k}\right)+\eta_{\rho_{i}, k}, \\
& v_{i, k+1}=v_{i, k}+\frac{\Delta t_{k}}{L_{i}} v_{i, k}\left(v_{i-1, k}-v_{i, k}\right)+ \\
& \frac{\Delta t_{k}}{\tau}\left\{v^{e}\left[\rho_{i, k}\right]-v_{i, k}\right\}-\frac{\nu \Delta t_{k}\left(\rho_{i+1, k}-\rho_{i, k}\right)}{\tau L_{i}\left(\rho_{i, k}+\kappa\right)}+\eta_{v_{i}, k}
\end{aligned}
$$

in which traffic is considered as a comprehensible fluid. $\tau$ denotes the relaxation constant, $\nu$ - the anticipation constant, $\kappa$ - a model parameter, preventing the third term in (17) to become zero [8], [7]. The flow in each section $i$ is presented as the average speed in that section times the average traffic density and the number of lanes in the section

$$
q_{i, k}=\rho_{i, k} v_{i, k} \ell_{i, k} .
$$

The average speed in equilibrium [7]

$$
v^{e}\left(\rho_{i, k}\right)=v_{f} \exp \left\{-\frac{1}{a_{m}}\left[\frac{\rho_{i, k}}{\rho_{i, c r i t}}\right]^{a_{m}}\right\}
$$

is computed based on the free-flow speed $v_{f}$ and the critical density $\rho_{i, c r i t}$ under the assumption that the vehicles do not influence each other. In order to guarantee numerical stability, the condition $v_{f} \Delta t_{k}<L_{i}$ must be respected [8]. Noises are added to the density and speed state variables and reflect modelling errors. The density noise $\eta_{\rho_{i, k}}$ and the speed noise $\eta_{v_{i}, k}$ are assumed to be Gaussian.

Figure 2 presents results obtained using the model (4)(13) for a traffic over 5 sections, with a congestion mode. In order to make comparison to the same states as in the stochastic METANET model, flow-density, speed-flow diagrams are plotted and the evolution of the flow and of the speed in time is presented. The state variable $Q_{i, k}$ from the compositional model has units $[v e h]$, whereas
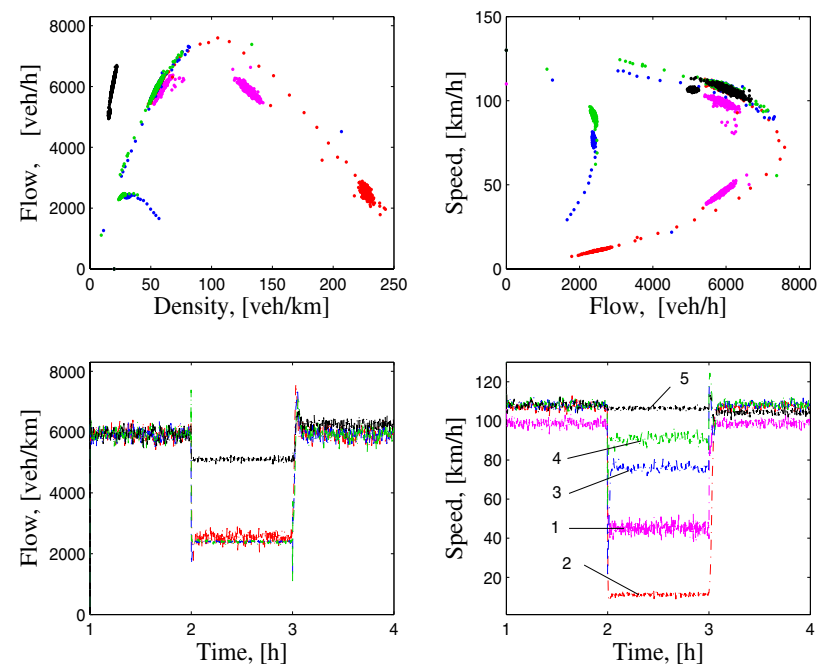

Fig. 2. Simulation results from the compositional model

$q_{i, k}$ from METANET is in $[v e h / h]$. The density and the flow are aggregated over all lanes of the freeway. An abrupt change occurs at $t_{k}=2[h]$. The number of lanes drops from 3 to 1 in sections 3 and 4 and hence until $t_{k}=3[h], \ell_{3, k}=\ell_{4, k}=1$.
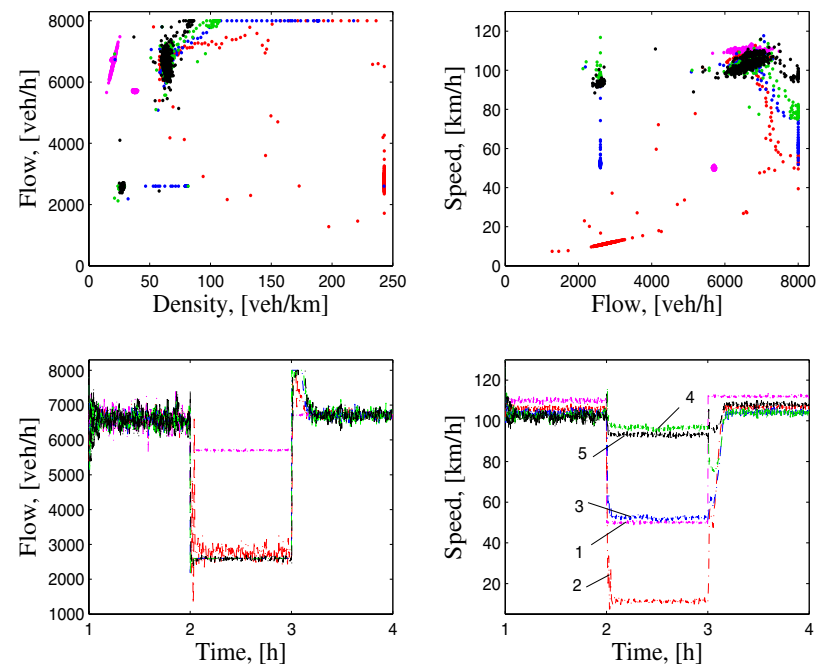

Fig. 3. Simulation results from the stochastic METANET model

For $t_{k}>3[h]$, the number of lanes is three again. The average vehicle length is assumed to be $A_{\ell}=0.01[\mathrm{~km}]$, the minimum time distance between vehicles $t_{d}=1[\mathrm{sec}]$, the free-flow speed is $v_{f}=130[\mathrm{~km} / \mathrm{h}]$, the minimum outflow speed $v_{\text {out }, \text { min }}=3[\mathrm{~km} / \mathrm{h}]$, the critical density $\rho_{i, \text { crit }}=32.5[\mathrm{veh} / \mathrm{km} /$ lane $]$. The coefficient values are chosen as follows: $\alpha=0.95, \beta=0.10$. Sampling is 
carried out with time step $\Delta t_{k}=10[\mathrm{sec}]=0.0028[h]$. The section length is $L_{i}=0.5[\mathrm{~km}]$. The speed noise covariance is $\operatorname{cov}\left(\eta_{v_{i}, k}\right)=0.5^{2}[\mathrm{~km} / \mathrm{h}]^{2}$, whereas the covariance of the sending function is non-stationary, equal to $\operatorname{cov}\left(\eta_{S_{i, k}}\right)=\left(0.03 N_{i, k} v_{i, k} \Delta t_{k} / L_{i}\right)^{2}[v e h]^{2}$. All noises are with Gaussian distribution.

The flow-density diagram from Fig. 2 shows that the compositional model describes well different traffic modes. In contrast to METANET, the compositional model does not require to preset the value of the density in jam. The mechanism of the sending and receiving functions affords an automatic change of the number of vehicles which can enter the next sections.

The stochastic METANET model (16)-(19) is applied to the same simulation scenario as the compositional model. Its parameters are chosen as follows: $\rho_{i, \text { crit }}=$ $32.5[\mathrm{veh} / \mathrm{km} /$ lane $], \tau=18[\mathrm{sec}], \nu=60\left[\mathrm{~km}^{2} / \mathrm{h}\right]$, $\rho_{\text {jam }}=80[\mathrm{veh} / \mathrm{km} /$ lane $], \kappa=40[\mathrm{veh} / \mathrm{km}], a_{m}=$ 1.867 , most of them such as in [7]. The capacity of the freeway (for all lanes) is $q_{\max _{i}, k}=8000,[v e h / h]$. The model errors are with covariances $\operatorname{cov}\left(\eta_{\rho_{i, k}}\right)=$ $0.12^{2}[\mathrm{veh} / \mathrm{km}]^{2}, \operatorname{cov}\left(\eta_{v_{i, k}}\right)=0.5^{2}[\mathrm{~km} / \mathrm{h}]^{2}$. The abrupt change of the number of lanes in sections 3 and 4 leads to a drop of the capacities to $q_{\max _{3}, k}=q_{\max _{4}, k}=$ $2600[v e h / h]$. After $t_{k}>3[h]$, the number of lanes is three again and $q_{\max _{3}, k}=q_{\max _{4}, k}=8000[\mathrm{veh} / \mathrm{h}]$. In the results obtained from the compositional model (Fig. 2) as well as from METANET (Fig. 3) one observes that the change in the number of lanes provokes a congestion with a backward wave: the congestion in lanes 3 and 4 is slowing down the drivers' speeds in sections 1 and 2 . Flow-density and flow-speed diagrams are presented in Fig. 3.

As seen from Figs. 2 and 3, both the compositional model and the stochastic METANET model describe very well the traffic phenomenon. The flow-density diagram has a bell-shaped form and the scattered zone in it (with density higher than the critical) represents the congestion when the number of the lanes is reduced.

\section{Conclusions}

A framework for modelling traffic on highways as a hybrid stochastic system is proposed. It is straightforward, general, and applicable to both freeways and urban networks, with different topologies, with any number of sensors, with regularly or irregularly received data in space and in time. The approach of modelling the traffic on freeways as a network of hybrid components has the following features: modularity, flexibility, suitable for parallel computations. Changes in the topology of the freeway network only require addition or deletion of a few components (local changes to the model). Its feasibility is demonstrated by the developed compositional traffic model and through a comparison to a stochastic METANET model. The compositional model is aimed at describing two types of traffic behavior: when the traffic flow propagates forward, i.e. from upstream to downstream sections (forward waves) and when the traffic flow propagates backward (upstream waves) in the presence of congestions. Results from simulation investigations are given. The compositional model can be applied to real traffic data for the goals of on-line traffic flow estimation, on-line control and modes detection.

\section{References}

[1] Daganzo, C., The Cell Transmission Model: A Dynamic Representation of Highway Traffic Consistent with the Hydrodynamic Theory, Transportation Research B, Vol. 28B, No. 4, pp. 269-287, 1994.

[2] Daganzo, C. F., A Finite Difference Approximation of the Kinematic Wave Model of Traffic Flow, Transportation Research B, Vol. 29B, No. 4, pp. 261-276, 1995.

[3] Gartner, N., C. Messer, and A. Rathi, Eds., Revised Monograph on Traffic Flow Theory, USA, 2002, http://www.tongji.edu.cn/ yangdy/its/tft/.

[4] Helbing, D., Traffic Data and Their Implications for Consistent Traffic Flow Modeling, in: M. Papageorgiou and A. Pouliezos (eds.) Transportation Systems, Vol. II (IFAC, Chania, Greece), pp. 809-814, 1997.

[5] Helbing, D., Traffic and Related Self-Driven Many-Particle Systems, Review of Modern Physics, Vol. 73, pp. 1067-1141, 2001.

[6] Hoogendoorn, S., and P. Bovy, State-of-the-art of Vehicular Traffic Flow Modelling, Special Issue on Road Traffic Modelling and Control of the Journal of Systems and Control Eng. Proc. of the IME I, 2001.

[7] Kotsialos, A., M. Papageorgiou, C. Diakaki, Y. Pavis, F. Middelham, Traffic Flow Modeling of Large-Scale Motorway Using the Macroscopic Modeling Tool METANET, IEEE Transactions on Intelligent Transportation Systems, Vol. 3, No. 4, pp. 282-292, 2002

[8] Papageorgiou, M., J. Blosseville, Macroscopic Model- ling of Traffic Flow on the Boulevard Périphérique in Paris, Transp. Research B, Vol. 23B, No. 1, pp. 29-47, 1989.

[9] Papageorgiou, M., and A. Kotsialos, Freeway Ramp Metering: an Overview, IEEE Transactions on Intelligent Transportation Systems, Vol. 3, No. 4, pp. 271-280, 2002.

Acknowledgments. Financial support by the project DWTC$\mathrm{CP} / 40$ "Sustainability effects of traffic management", Belgium is gratefully acknowledged, and also by the Programme on InterUniversity Poles of Attraction initiated by the Belgian State, Prime Minister's Office for Science, Technology and Culture, and in part by the Bulgarian Foundation for Scientific Investigations under grants I-1202/02 and I-1205/02. We also thank the "Vlaams Verkeerscentrum Antwerpen", Antwerp, Belgium, and Mr. Frans Middleham from the Transport Research Centre of the Ministry of the Transport, the Netherlands for providing the data used in this study. 